\title{
Correction to: Density functional theory studies on PVDF/ionic liquid composite systems
}

\author{
RANJINI SARKAR and T K KUNDU* \\ Department of Metallurgical and Materials Engineering, Indian Institute of Technology Kharagpur, Kharagpur, \\ West Bengal 721 302, India \\ E-mail: tkkundu@metal.iitkgp.ac.in \\ Published online 3 December 2018
}

\section{Correction to:}

\section{J. Chem. Sci. (2018) 130:115 https://doi.org/10.1007/s12039-018-1522-4}

The term Hirshfeld was spelt incorrectly in the published article. The corrected sentences read as follows:

Abstract: Mulliken and atomic dipole moment corrected Hirshfeld population analyses were carried out to provide a quantitative analysis of partial atomic charge distribution.

$4^{\text {th }}$ paragraph under section 2 Computational details: Partial atomic charge distribution within the systems under study is demonstrated using two types of charge population analysis schemes, a) Mulliken population analysis (MPA) and b) Hirshfeld population analysis (HPA). MPA $^{40}$ provides a means of estimating partial atomic charges from calculations carried out by the methods involving a linear combination of atomic orbital molecular orbital (LCAO MO) theory. However, the major disadvantage of this population analysis scheme is its high basis set dependence and therefore less reliability compared to HPA method, which is based on deformation density partition. ${ }^{41}$ But Hirshfeld atomic charges are too small and have a poor reproducibility of the observable quantities as it ignores atomic dipole moments. Therefore, atomic dipole moment corrected Hirshfeld $(\mathrm{ADCH})$ population analysis ${ }^{42}$ is also carried out, where Hirshfeld charges are corrected by expanding atomic dipole moments to correct charges placed

\footnotetext{
*For correspondence

The original article can be found online at https://doi.org/10. 1007/s12039-018-1522-4.
}

at neighbouring atoms. In the current study, HPA and ADCHPA are carried out in Multiwfn program. ${ }^{43}$

$2^{\text {nd }}$ sentence under section 3.5 Frontier orbitals, their composition analysis and chemical parameters:

The HOMO and LUMO compositions of all the atoms present in the molecules are calculated with Hirshfeld orbital composition analysis ${ }^{55}$ algorithm using Multiwfn program. ${ }^{43}$

Table 5 caption:

Table 5. HOMO and LUMO compositions of pure $\beta$ PVDF and $\beta$-PVDF/IL systems, calculated using Hirshfeld orbital composition analysis algorithm in Multiwfn (atom numbers are given according to Figure 3(b)) [Calculation method: DFT-D; functional: B3LYP; basis set: 6-311+G(d,p)].

$8^{\text {th }}$ sentence under section 4 Conclusions:

Mulliken population analysis (MPA) and atomic dipole moment corrected Hirshfeld (ADCH) population analysis have been carried out to quantify the charge distribution within all the systems under study.

Apart from the term Hirshfeld, some of the text under section 3.4 Population analysis was published incorrectly. The corrected paragraph should read as follows:

\subsection{Population analysis}

Mulliken and Hirshfeld population analyses have been carried out for a pristine $\beta$-PVDF molecule using different functional and basis sets to provide a comparative description of the two population analysis schemes (Table S3, Supplementary Information). As mentioned in Section 2, HPA is found to be basis set independent. The change in atomic dipole moment corrected Hirshfeld (ADCH) charge distribution within $\beta$-PVDF molecule after the ionic liquid addition is shown in Table 4 (Complete $\mathrm{ADCH}$ population analysis performed for 
all $\beta$-PVDF/IL systems under study is presented in Table S4, Supplementary Information). Within the $\beta$-PVDF molecule, all the fluorine atoms $(\mathrm{F} 9-16)^{\$}$ and the carbon atoms bonding with the hydrogen atom pairs $(\mathrm{C} 1$, $\mathrm{C} 3, \mathrm{C} 5, \mathrm{C} 7)^{\$}$ contain negative partial charges, i.e., they are electron acceptors. On the other hand, all the hydrogen atoms (H17-26) ${ }^{\$}$ and the carbon atoms bonding with fluorine atom pairs possess $(\mathrm{C} 2, \mathrm{C} 4, \mathrm{C} 6, \mathrm{C} 8)^{\$}$ positive atomic charges and act as electron donors (Table 4). This explains the higher electronegativity of $\mathrm{F}$ atoms in $\mathrm{C}-\mathrm{F}$ bonds and lower electronegativity of $\mathrm{H}$ atoms in $\mathrm{C}-\mathrm{H}$ bonds. No significant change in population is observed within $\beta$-PVDF part of PVDF/IL complexes with the change in alkyl chain length of the IL cation. But a larger variation on a charge is observed in the ionic liquid portion of the complex molecules, suggesting higher chemical reactivity of ILs. The highest positive charge is accumulated within the boron atom $\left(\mathrm{B} 27^{\$}\right)$ present in the $\left[\mathrm{BF}_{4}\right] \mathrm{IL}$ anion and $\mathrm{H} 42^{\$}, \mathrm{H}_{4} 3^{\$}$ atoms in the IL cation. The highest negative charge is found to be accumulated within the fluorine atoms present in the anion and the $\mathrm{C} 36^{\$}, \mathrm{C} 37^{\$}$ atoms (Table S4, Supplementary Information) within IL cation ( $\$$ refer to Figures 1 and 3 (b) for atom numbers). 